\section{International Scientific Journal Theoretical \& Applied Science}

Dmitry Nikolayevich Chernov Associate professor, candidate of psychological sciences, Professor of the Department of general psychology and pedagogic,

Pirogov Russian National Research Medical University, Moscow, Russia chernov_dima@mail.ru

Year: 2017 Issue: $06 \quad$ Volume: 50

Published: $24.06 .2017 \quad$ http://T-Science.org

SECTION 21. Pedagogy. Psychology. Innovations in the field of education.

\title{
THE PROBLEM OF STUDYING OF THE PERSONALITY TRAITS' EXPRESSION IN THE ACOUSTIC CHARACTERISTICS OF SPEECH
}

Abstract: This paper analyzes the results of studies of the acoustic correlates of individual psychological characteristics of personality. The experimental problems that must be solved in order to create an integrated model of the personality traits' expression in the acoustic characteristics of speech are discussed. The possible physiological mechanisms of the manifestation of personality characteristics in the speech signal are considered.

Key words: acoustic characteristics, voice, personality, personality traits, speech, speech signal.

Language: Russian

Citation: Chernov DN (2017) THE PROBLEM OF STUDYING OF THE PERSONALITY TRAITS' EXPRESSION IN THE ACOUSTIC CHARACTERISTICS OF SPEECH. ISJ Theoretical \& Applied Science, 06 (50): 82-86.
Soi: http://s-o-i.org/1.1/TAS-06-50-9
Doi: crossef https://dx.doi.org/10.15863/TAS.2017.06.50.9

\section{ПРОБЛЕМА ИССЛЕДОВАНИЯ ВЫРАЖЕНИЯ СВОЙСТВ ЛИЧНОСТИ В АКУСТИЧЕСКИХ ХАРАКТЕРИСТИКАХ РЕЧИ}

Аннотация: В работе проанализированы результаты исследований акустических коррелятов индивидуально-психологических особенностей личности. Обсуждаются экспериментальные задачи, которые необходимо решить для того, чтобы построить интегральную модель выражения личностных свойств в акустических характеристиках речи. Рассмотрены возможные психофизиологические механизмы проявления личностных особенностей в речевом сигнале.

Ключевые слова: акустические характеристики, голос, личность, личностные свойства, речь, речевой сигнал.

\section{Introduction}

Многочисленные экспериментальные исследования, выполненные в контексте изучения психофизиологических и психологических основ невербального общения, показывают, что кроме лингвистической информации речевой сигнал является носителем самого разнообразного содержания. Через слуховой канал можно получить эмоциональную, эстетическую, индивидуально-личностную, биофизическую, социально-типологическую, пространственную, психологическую и медицинскую информацию о говорящем, информацию о физических помехах, сопутствующих речепорождению [16]. В настоящей работе мы попытаемся понять, существуют ли объективные акустические корреляты устойчивых индивидуальнопсихологических особенностей личности?

\section{Materials and Methods}

Проблемы выражения в речи различных функциональных и эмоциональных состояний давно интересуют ученых. Основы этого направления исследований, по-видимому, были заложены Ч. Дарвином (1872) в работе «О выражении эмоций у человека и животных» [10]. Экспериментальное развитие акустический подход к изучению психоэмоциональных состояний получил в связи с разработкой акустической теории речеобразования $[11 ; 13]$. В России практическая востребованность исследований речи стали причиной проведения симпозиумов «Речь и эмоции» (1974) и «Речь, эмоции и личность» (1978) и совещаний по акустике речи и слуха (АРСО). Было обнаружено, что информативными акустическими коррелятами психоэмоциональных состояний являются: показатели (среднее, максимальное и 
минимальное значение, дисперсия, диапазон) частоты основного тона $\left(F_{0}\right)$, формантных частот $\left(F_{1}, F_{2}, . . F_{\text {n }}\right)$ и ширины формантных зон $\left(B_{1}, B_{2}, .\right.$. $\left.B_{\mathrm{n}}\right)$, огибающей спектра, интенсивности речи $(I)$, распределения низко- и высокочастотной энергии в спектре, формы интонационного контура высказывания. Востребованными оказались временные характеристики: латентное время ответной реакции, длительность и темп речи, длительность $(t)$ пауз, ударных гласных, слогов и фраз. В целом, исследования демонстрируют, что стенические состояния ведут к возрастанию, а астенические - к понижению показателей $F_{0}, F_{\mathrm{n}}$ и I. Эта связь обусловлена индивидуальными особенностями говорящих: половыми различиями, степенью экстра/интроверсии, уровнем нейротизма и тормозным/возбудимым типом реагирования [1]. Вместе с тем, обнаружено, что при возникновении различных психоэмоциональных состояний показатели $F_{0}, I$ и темпа речи изменяются взаимосвязано. Было бы неверно без тщательной экспериментальной проверки опираться на каждую из указанных выше характеристик голоса в отдельности как на показатель, обладающий дифференцирующей силой [4].

Изучению акустических коррелятов свойств темперамента и личности посвящены единичные исследования. Например, на русскоязычной выборке мужчин А.В. Никоновым и Е.В. Беловол (2000) обнаружено, что лица, относящиеся к разным типам темперамента, отличаются по $F_{0}$ и ее производным; эмоциональность прямо, а эргичность (желание умственного и физического напряжения, избыток сил) - обратно пропорционально связаны с показателями $F_{0}$ [17]. Вопрос о механизмах обнаруженных связей, как правило, остается за рамками подобных исследований. Вопрос о создании целостной концепции проявлений психологической индивидуальности в параметрах голоса на сегодняшний день по-прежнему открыт. Разработка модели диагностики разноуровневых психологических особенностей по акустическим параметрам речи позволила бы: проводить комплексную психодиагностику индивидуальности; сократить временные и энергетические затраты на осуществление индивидуально-психологического обследования; расширить диапазон применения психологического тестирования (распространить на ситуации, когда использование бланковых психодиагностических методик не возможно или не рекомендуется, например, в клинической практике: при отсутствии мотивации к обследованию, высокой вероятности проявления социальной желательности в ответах обследуемого и т.п.). Для построения такой интегральной модели требуется решить ряд задач [6].

Bo-nервых, необходимо определиться с акустическими характеристиками речи, которые должны быть выделены из речевого сигнала, обеспечить их адекватную регистрацию и обработку. Эта проблема хорошо разработана в контексте акустической теории речеобразования $[11 ; 13]$ и прикладной фонетики [22].

Bo-вторых, необходимо установить степень межиндивидуальной вариативности акустических характеристик голоса. Малая изменчивость того или иного акустического параметра не позволяет рассматривать его в качестве индивидуальной характеристики голоса человека. Этому вопросу уделялось значительно меньше внимания. Показано, что ряд акустических характеристик обладает существенной межиндивидуальной изменчивостью $[5 ; 7 ; 8 ; 9 ; 12]$. Возможно выделение типологических акустических особенностей в зависимости от характера речевой деятельности [15].

B-третьих, необходимо выяснить внутрииндивидуальную стабильность выделенных параметров. Этот вопрос оказался наименее разработанным. В ряде исследований обнаружена внутрииндивидуальная стабильность показателей $F_{0}$ при произнесении разнородного языкового материала [2], показателей $F_{0}-F_{4}$ и $B_{1}-$ $B_{4}$ при произнесении слогов $[7 ; 8]$, чтении текста [9] и свободном рассказе [5]. Стабильность акустических характеристик при изложении языкового материала в иных (экспериментальных и приближенных к реальной жизнедеятельности) контекстах требует экспериментальной проверки.

Четвертым вопросом, дополнительным для решения рассматриваемой проблемы, является изучение вклада наследственных и средовых факторов в формирование индивидуальных особенностей голоса. На сегодняшний день в силу различных методических причин этот вопрос открыт [3]. На русскоязычной выборке близнецов показано, что индивидуальные показатели $F_{0}$ и $F_{1}-F_{3}$ находятся под воздействием факторов наследственности и индивидуальной среды; умеренные общесредовые влияния на формирование индивидуальных различий по средним значениям $F_{2}$ и $F_{3}$ объясняются фонетическими особенностями произносимого материала [8]. Основываясь на знании о генотип-средовых соотношениях различных индивидуальнопсихологических особенностей человека и акустических параметров речи, окажется возможным определить пределы корреляций между психологическими и акустическими показателями и выдвинуть предположения о механизме этих связей. 
Только после решения этих задач можно переходить к изучению пятого вопроса выявлению акустических коррелятов устойчивых личностных характеристик. Таких исследований - единицы. Кроме упомянутой выше работы А.В. Никонова и Е.В. Беловол (2000), на выборке русскоязычных испытуемых, с учетом всех обсуждаемых в данном разделе проблем, выполнено еще несколько исследований. Они показали, что некоторые средние и показатели внутрииндивидуальной вариативности $F_{0}, F_{2}, F_{3}$, $B_{1}$ и $B_{3}$ могут быть маркерами представленности в структуре личности (по данным опросника MMPI) компонентов: мужественностьженственность, психопатия, гипомания, психастения, ипохондрия и истерия [5;9].

Выявление

механизма реализации индивидуальнопсихологических свойств личности в акустических характеристиках голоса является шестой (и наиболее сложной!) задачей, которая должна быть изучена в контексте рассматриваемой темы. Рассмотрим этот вопрос подробнее. Существует несколько возможных психофизиологических механизмов выражения личностных особенностей в акустических показателях речи.

1) Наиболее психологических наблюдаться с $F_{0}$. Средняя $F_{0}$ обычно соответствует привычной для индивида частоте колебания голосовых связок. Исследования, проведенные в русле генетики поведения, наглядно демонстрируют, что вклад наследственных факторов в индивидуальные различия по $F_{0}$ составляет примерно $50 \%$. А значит, остальная часть фенотипической дисперсии по средним значениям $F_{0}$ обусловлена ситуативными или долговременными средовыми влияниями [8]. В качестве одного из таких средовых факторов, например, можно рассматривать нетипичный для индивида уровень напряжения голосовых связок вследствие какоголибо переживаемого психического состояния, что приводит к повышению «базовой» $F_{0}$. Например, предполагалось, что у депрессивных больных $F_{0}$ ниже, чем у здоровых индивидов. Однако обнаружено, что в среднем $F_{0}$ у таких больных не отличается от нормы. При этом депрессивные больные говорят тихо в силу низкого уровня подглоттального давления. Иными словами, чтобы сохранить среднюю $F_{0}$ на уровне нормы, больным приходится чрезмерно напрягать голосовые связки [18]. К сожалению, $F_{0}$ является не лучшим акустическим показателем устойчивых индивидуально-психологических особенностей человека, поскольку можно предположить, что дикторы способны произвольно контролировать изменения этой характеристики в процессе речепроизводства.

2) Индивидуально-личностные особенности могут выражаться в устойчивых характеристиках $F$-картины. Относительное расположение энергетических пиков, соответствующих частоте основного тона и формантным частотам $\left(F_{1}-F_{0}\right.$, $\left.F_{2}-F_{1}, \ldots F_{\mathrm{n}}-\mathrm{F}_{\mathrm{n}-1}\right)$, позволяет нам, независимо от присущих речи диктора устойчивых особенностей расположения формант, опознавать отдельные фонемы. $F_{\mathrm{n}}$ с такой функцией выдающийся российский ученый Н.И. Жинкин (1958) называл речевыми формантами; они определены нормами языка. Физиологическим обеспечением сдвига $F_{\text {n }}$ относительно друг друга являются ситуативные изменения длины вокального тракта. Абсолютные устойчивые частотные характеристики формант $\left(F_{\mathrm{n}}\right)$ конкретного диктора, зависящие от конфигурации его голосового тракта, создают специфическую тембровую окраску речи индивида и, соответственно, позволяют опознать его по голосу. Такие $F_{\mathrm{n}}$, которые могут быть вычислены посредством усреднения значений по каждой из формант, полученных в зависимости от специфики конкретных звуков, были названы голосовыми формантами [21]. Голосовые форманты обусловлены анатомическими особенностями строения голосового тракта. Однако данные исследований, проведенных в русле генетики поведения, указывают на существенную средовую обусловленность индивидуальных особенностей $F_{\mathrm{n}}[7 ; 8]$. Так, в качестве фактора, создающего средовую вариативность $F_{\mathrm{n}}$, можно расценивать произвольные изменения $F$-картины посредством манипуляций с артикуляционным аппаратом: например, продвижение языка вперед, подъем нижней челюсти и гортани способствует уменьшению длины вокального тракта и, как следствие, ведет к повышению формант. Эти приемы используются специалистами в работе с мужчинами, меняющими сексуальную ориентацию в целях приближения в речи к стандартам женственности [19]. Очевидно, что именно особенности голосовых формант человека могут быть коррелятами его психологических характеристик. Тем не менее, разности $F_{\mathrm{n}}-\mathrm{F}_{\mathrm{n}-1}$ также могут нести важную психологическую нагрузку. При отклонении разностей между формантами от предполагаемых в фонетике канонических величин, фонемы становятся мало различимы на слух, гласные звуки начинают сливаться. Такие устойчивые особенности говорения («вялая» артикуляция) могут выражать отсутствие коммуникативной цели быть понятым окружающими, что, в свою очередь, может отражать устойчивые личностные особенности индивида. 
3) В качестве одного из коррелятов индивидуально-психологических особенностей человека может выступать относительная концентрация энергии в низко- (Lower Frequency Region, LFR) и высокочастотной (Higher Frequency Region, HFR) областях спектра. Увеличение энергии в LFR (обычно рассчитывается по области ниже 1000 Гय) наблюдается при общем снижении мышечного напряжения, что должно отражаться и на расслаблении мышц, задействованных в процессе речепроизводства. Данный феномен обнаружен при изучении влияния психотерапевтических (на примере психических заболеваний) и медикаментозных воздействий (на примере группы нормы) на мышечное напряжение, что одновременно коррелировало с увеличением доли спектральной энергии в LFR. Напротив, концентрация энергетического максимума в $H F R$, отмечается при общем повышении мышечного напряжения [14; 20]. Тогда устойчиво высокая доля энергии в HFR будет указывать на то, что индивид находится в состоянии постоянного психоэмоционального и физического напряжения и наоборот. Обзор работ, касающихся выражения в акустических показателях речи психоэмоциональных состояний, показывает, что при возникновении тех или иных состояний наблюдаются взаимосвязанные изменения $B_{\mathrm{n}}$ и доли спектральной энергии в LFR или HFR [4]. Таким образом, в основе связей $B_{1}$ со шкалой психастении и $B_{3}$ со шкалой гипомании (по данным опросника $M M P I)$, обнаруженных в исследовании Д.Н. Чернова с соавт. (2008), могут лежать изменения относительной доли энергии в $L F R$ или HFR. Физиологической основой этих связей может быть пониженный или повышенный общий тонус мышц и степень напряженности мышц, задействованных в процессе речепроизводства, в частности. Впрочем, эти предположения требуют дополнительной экспериментальной проверки.

\section{Conclusion}

Таким образом, изучению психофизиологических механизмов реализации личностных особенностей человека в его голосе должны быть посвящены специальные психофизиологические исследования, что требует интеграции усилий специалистов в области психологии, физиологии и акустики речи.

\section{References:}

1. Adashinskaja GA, Chernov DN (2007) Akusticheskie korreljaty individual'nyh osobennostej funkcional'nyh i jemocional'nyh sostojanij // Aviakosmicheskaja i jekologicheskaja medicina. - T. 41. - № 2. - p. 3-13.

2. Belovol EV (1999) Projavlenie svojstv temperamenta $\mathrm{v}$ akusticheskih harakteristikah rechi. Dis. ... kand. psihol. nauk. - M..

3. Chernov DN (2005) Psihogeneticheskie issledovanija individual'nyh osobennostej golosa: aktual'noe sostojanie i perspektivy / XX Merlinskie chtenija: «V.S. Merlin i sistemnye issledovanija individual'nosti cheloveka»: Materialy mezhreg. jubilejnoj nauch.-prakt. konf.. Permskij gos. ped. un-t, may 19-20, 2005. Perm' / Pod red. B.A. Vjatkina, A.A. Volochkova. V 3 chastjah. - Ch. II. - Perm'. p. 51-54.

4. Chernov DN, Ignatov JJ (2008) Golosovye korreljaty psihojemocional'nyh sostojanij: metaanaliz obzornyh rabot / Materialy 1 Vseross. nauch.-prakt. konf. «Psihologija psihicheskih sostojanij: teorija i praktika», g. Kazan', Kazanskij gos. un-t, november 13-15,
2008. - Ch. 1. - Kazan': ZAO «Novoe znanie», - p. 460-463.

5. Chernov DN, Ignatov JJ, Nigorodova VS, Parshukov AJ (2008) Vyrazhenie lichnostnyh osobennostej cheloveka $\mathrm{V}$ ego golose / Materialy Mezhd. teoret. i nauch.-prakt. konf. «Social'no-jekonomicheskie i gumanisticheskie osnovy obnovljajushhejsja Rossii», 24-25 aprelja 2008 g. Mezhd. slavjanskij in-t, Mezhd. Kirillo-Mefodievskaja akad. slavjanskogo prosveshhenija i dr. - M.: OOO «Diona». - p. 221-226.

6. Chernov DN, Parshukov AJ (2008) Aktual'nye problemy izuchenija akusticheskih korreljatov psihologicheskih svojstv individual'nosti / Obshhestvo - Jazyk - Kul'tura: aktual'nye problemy vzaimodejstvija v XXI veke: Doklady Mezhd. nauch.-prakt. konf. Moskovskij in-t lingvistiki, g. Moscow, october 18, 2007. - T. 1. - M.: Petrorush. - p. 176-181.

7. Chernov DN, Parshukov AJ (2006) Genotipsredovye sootnoshenija po akusticheskim harakteristikam golosa / Vtoraja mezhd. konf. po kognitivnoj nauke: Tez. dokladov: $\mathrm{V} 2 \mathrm{t}$. Sankt-Peterburg, june 9-13, 2006. - SPb.: 


\begin{tabular}{l|lrl|l|ll} 
& ISRA (India) & $=\mathbf{1 . 3 4 4}$ & SIS (USA) & $=\mathbf{0 . 9 1 2}$ & ICV (Poland) & $=\mathbf{6 . 6 3 0}$ \\
Impact Factor: & ISI (Dubai, UAE) $=\mathbf{0 . 8 2 9}$ & PUHL (Russia) $=\mathbf{0 . 2 3 4}$ & PIF (India) & $=\mathbf{1 . 9 4 0}$ \\
& GIF (Australia) & $\mathbf{0 . 5 6 4}$ & ESJI (KZ) & $=3.860$ & IBI (India) & $=\mathbf{4 . 2 6 0}$ \\
& JIF & $\mathbf{1 . 5 0 0}$ & SJIF (Morocco) & $=\mathbf{2 . 0 3 1}$ & & \\
\hline
\end{tabular}

Filologicheskij fakul'tet SPbGU. - T. 2. - p. 474-475.

8. Chernov DN, Parshukov AJ (2006) Jeksperimental'noe issledovanie genotipsredovyh sootnoshenij po akusticheskim harakteristikam rechi // Psihologicheskij zhurnal. - T. 27. - № 4. - p. 86-94.

9. Chernov DN, Parshukov AJ, Ignatov JJ, Nigorodova VS (2008) Jeksperimental'noe issledovanie projavlenija osobennostej lichnosti cheloveka $\mathrm{v}$ akusticheskih pokazateljah ego rechi / Materialy mezhd. nauch. konf. «Informacija, signaly, sistemy: voprosy metodologii, analiza i sinteza». Tehnologicheskij in-t Juzhnogo Federal'nogo un-ta, g. Taganrog, may, 2008. - Ch. 2. Taganrog: Izd-vo TTI JuFU, - p. 77-81.

10. Darvin C (2001) O vyrazhenii jemocij u cheloveka i zhivotnyh. - SPb.: Piter.

11. Fant G (1964) Akusticheskaja teorija recheobrazovanija. - M.: Nauka.

12. Fant G, Kruckenberg A, Liljencrants J, Botinis A (2002) Individual variations in prominence correlates. Some observations from «labspeech» // TMH-QPSR. - Vol. 44. - № 1. - p. 177-180.

13. Flanagan D (1968) Analiz, sintez i vosprijatie rechi. - M.: Mir.

14. Helfrich H, Standke R, Scherer KR (1984) Vocal indicators of psychoactive drug effects // Speech Communication. - Vol. 3. - № 3. - p. 245-252.
15. Holm S (2003) Individual use of acoustic parameters in read and spontaneous speech // Phonum. - Vol. 9. - № 1. - p. 157-160.

16. Morozov VP (2003) Neverbal'naja kommunikacija $\mathrm{V}$ sisteme rechevogo obshhenija: psihofiziologicheskie i psihoakusticheskie osnovy / Psihologija XXI veka / Pod red. V.N. Druzhinina. - M.: PER SJe, - p. 415-440.

17. Nikonov AV, Belovol EV (2000) Sootnoshenie formal'no-dinamicheskih svojstv individual'nosti $\mathrm{i}$ akusticheskih harakteristik rechi // Psihologicheskij zhurnal. - T. 21. - № 5. - p. 65-69.

18. Scherer K (19797) Nonlinguistic vocal indicators of emotion and psychopathology / in C.E. Izard (Ed.) Emotions in personality and psychopathology. - N.-Y.: Plenum Press. - p. 495-529.

19. Smyth R, Jacobs G, Rogers H (2003) Male voices and perceived sexual orientation: an experimental and theoretical approach // Language and Society. - Vol. 32. - № 3. - p. 329-350.

20. Tolkmitt FJ, Helfrich H, Standke R, Scherer KR (1982) Vocal indicators of psychiatric treatment effects in depressives and schizophrenics // Journal of Communication Disorders. - Vol. 15. - № 3. - p. 209-222.

21. Zhinkin NI (1958) Mehanizmy rechi. - M.: Izdvo APN.

22. Zlatoustova LV, Potapova RK, Potapov VV, Trunin-Donskoj NV (1997) Obshhaja i prikladnaja fonetika. - M.: Izd-vo MGU. 